\title{
FIR photoconductivity at filamentary current flow in n-GaAs in a magnetic field
}

\author{
V.G. Golubev \\ A.F. Ioffe Physico-Technical Institute, Academy of Sciences, Leningrad, USSR \\ W. Prettl \\ Institut für Angewandte Physik, Universität Regensburg, FRG
}

(Received May 17, 1991 by M. Cardona)

\begin{abstract}
A large number of spectral structures are observed by far infrared magneto-photoconductivity in $\mathrm{n}$-GaAs at $4.2 \mathrm{~K}$ biased above impurity breakdown in a regime where current flow is filamentary. Phase changes are found in the center of the $1 s-2 p_{+1}$ line and new structures are observed which shift to higher magnetic fields with increasing bias voltage. The large strength and the intensity dependence of the photosignals indicate that the observed phenomena are due to self-organizing effects of the filament. Small variations of the electron population by infrared irradiation may cause substantial rearrangements of the spatial current distribution.
\end{abstract}

At low temperatures the autocatalytic process of impact ionization of shallow impurities in high-purity semiconductors leads to highly nonlinear current-voltage characteristics. At a critical breakdown voltage the impact ionization rate of impurities exceeds the capture rate of free carriers at low concentrations yielding a rapid increase of the current at a practically constant voltage across the sample. At breakdown a strongly ionized channel is formed in the sample constituting a current filament which carries the total current. The increase of the current is then caused by a lateral growth of the current filament.

Here we report on peculiarities which were found in the photoconductivity of $n-G a A s$ when the sample was biased in the nonlinear regime of the current-voltage characteristic and subjected to an external magnetic field. Enhancement of resonant structures in magneto-photoconductivity spectra due to free carrier multiplication, resonant negative photoconductivity and even structures which cannot be attributed to optical impurity transitions are observed. The phenomena may qualitatively be understood from the spatial structure of the filamentary current flow in n-GaAs [1] and the Hall voltage which develops across the filament in a magnetic field leading to different free carrier multiplication rates at opposite edges of the filament [2].

The measurements were carried out on $\mathbf{n}-\mathrm{GaAs}$ epitaxial layers prepared on semiinsulating substrates by vapour phase epitaxy. Results are presented for a sample of $N_{D}-N_{A}=2.7 \cdot 10^{14} \mathrm{~cm}^{-3}$ effective donor concentration, $N_{A} / N_{D}=0.35$ compensation ratio and $14 \mu \mathrm{m}$ thickness. The size of the sample was $3 \times 5 \mathrm{~mm}^{2}$; ohmic Indium point contacts were alloyed on opposite edges along the shorter side. The sample was immersed in liquid helium at $4.2 \mathrm{~K}$ in the center of a superconducting solenoid. As sketched in the insert of Fig. 2, the epitaxial layer showed the typical nonlinear currentvoltage relation with three characteristic regimes: (1) high-ohmic pre-breakdown current flow, (2) transition regime from high-resistive to low-resistive behaviour and (3) low-ohmic stable post-breakdown regime. The photoconductive response in Faraday configuration was measured for various biasing conditions as a function of the magnetic field strength up to $6 \mathrm{~T}$ using a standard load resistor circuit and lock-in technique. As radiation sources an $\mathrm{H}_{2} \mathrm{O}$ and an $\mathrm{HCN}$ laser, both $\mathrm{cw}$ electrically pumped, were applied with about $10 \mathrm{~mW}$ continuous output power. Two laser lines were used with wavelengths $\lambda=118 \mu \mathrm{m}$ and $337 \mu \mathrm{m}$ corresponding to photon energies larger and smaller, respectively, than the binding energy of shallow donors at zero magnetic field. Taking into account reflection losses at the sample surface, the maximum intensity in the epitaxial film was estimated to be about $25 \mathrm{~mW} \cdot \mathrm{cm}^{-2}$.

Fig. 1 shows measurements of the photoconductive signal for various constant bias currents at $\lambda=$ $118 \mu \mathrm{m}$. All identified spectral structures may be attributed to optical excitations from the donor ground state to excited states. The observed transitions are labelled by the quantum numbers of the final states. Magnetic field quantum numbers $(N, m, k)$ are used where $N$ is the Landau level quantum number, $m$ the angular momentum parallel to the magnetic field and $k$ counts the state below each Landau sublevel $(N, m)$. Final bound states are also indicated by hydrogen atom quantum numbers $n l_{m}$. The lowest trace in the figure shows a recording where the sample was biased in the pre-breakdown regime ((1) in the insert of Fig. 2). 


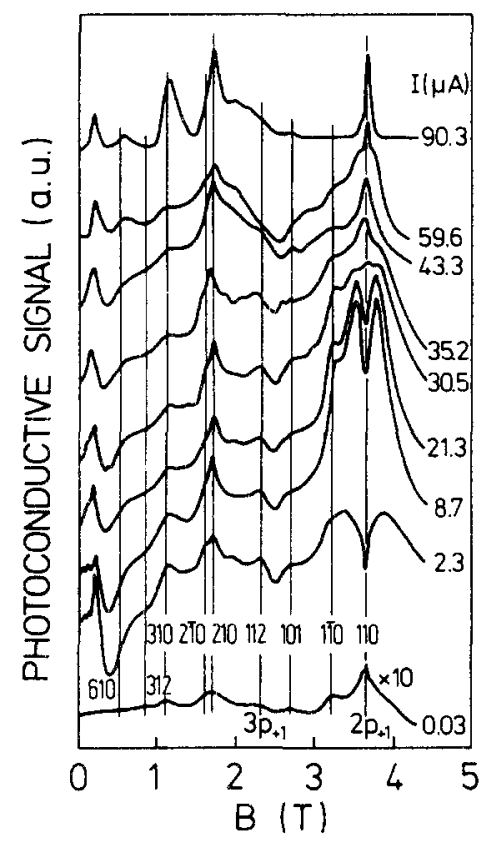

Fig. 1

Photoresponse as function of magnetic field at $\lambda=$ $118 \mu \mathrm{m}$ for various constant bias currents.

Besides the $1 s-2 p_{+1}$ and $1 s-3 p_{+1}$ photoconductivity lines, resonant structures due to optical transitions to excited metastable states with $m<N$ are observed. In atom-like systems subjected to a magnetic field metastable states grow out of the continuum in form of resonances $[3,4]$. With increasing bias voltage a strong enhancement of the photosignal due to resonances was found and pronounced changes in the line shape including a change of the phase in the center of the $1 s-2 p_{+1}$ line were observed.

Even more drastic changes in the spectra occur in the post-breakdown regime (3) as shown in Fig. 2 . The transitions to metastable states disappear and new spectral structures show up which in most cases shift to higher magnetic fields with increasing voltage (Fig. 2a). Different signal peaks move differently with bias voltage. Similar structures in the spectra were observed at $\lambda=337 \mu \mathrm{m}$ (Fig. 2b) for the photon energy being smaller than the binding energy of shallow donors at $B=0$. We point out that even weak spectral structures in Fig. 2 are real and are reproducible with high signal to noise ratio. The observed signals differ in strength by more than three orders in magnitude.

In the breakdown and post-breakdown regime $1 s-$ $2 p_{+1}$ (Fig. 1 and Fig. 2a) transitions and the cyclotron resonance of free electrons (Fig. 2b) are observable for any biasing condition. However, most of the new photoconductivity structures are restricted to certain ranges of bias voltage and magnetic field strength. The shape of the peaks in regime 3 of the current-voltage characteristic depends strongly on the bias voltage. Even the phase of the signal may be inverted in a very nar-

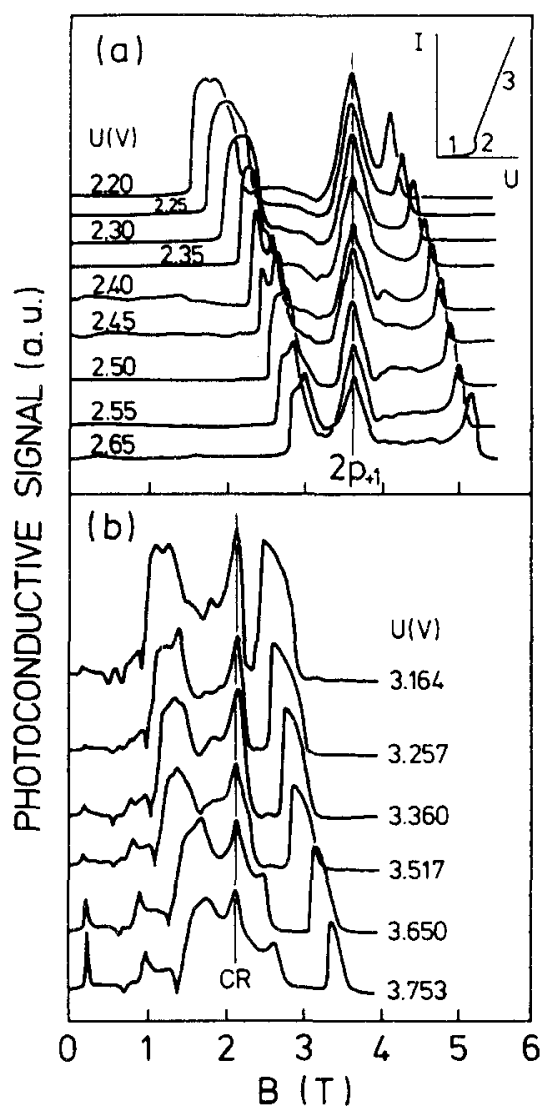

Fig. 2

Photoresponse as function of magnetic field $B$ for different bias voltages. Bias voltages are given for $B=0$; for constant external voltage across the series combination of load resistor and sample the bias voltage increases ( 2 to 3 times) with rising magnetic field. (a) $\lambda=118.6 \mu \mathrm{m}$; (b) $\lambda=337 \mu \mathrm{m}$. Inset shows typical current-voltage characteristic.

row voltage range. The width of different peaks may be very different varying from about $10 \mathrm{mT}$ (Fig. 3a) up to several Tesla. The observed structures depend also on interband illumination and on the orientation of the magnetic field with respect to the direction of current flow. Fig. 3b shows the dependence of the photoresponse on far-infrared irradiation intensity. Curve 1 and 3 are in the regime of linear response. With rising intensity the observed structures widen on the magnetic field scale (curve 2) and even new photoconductivity lines may arise if the intensity surpasses a threshold value (curve 4). The threshold intensities depend on the magnetic field and the bias voltage and are typically in the range of a few $\mathrm{mW} \cdot \mathrm{cm}^{-2}$.

The observed phenomena are caused by nonlinear free carrier generation and recombination processes due to impact ionization of shallow donors. Impact ionization of donors in excited states yields the growth of the photoconductive signals of resonant transitions with rising bias current shown in Fig. 1. These photo-impact 


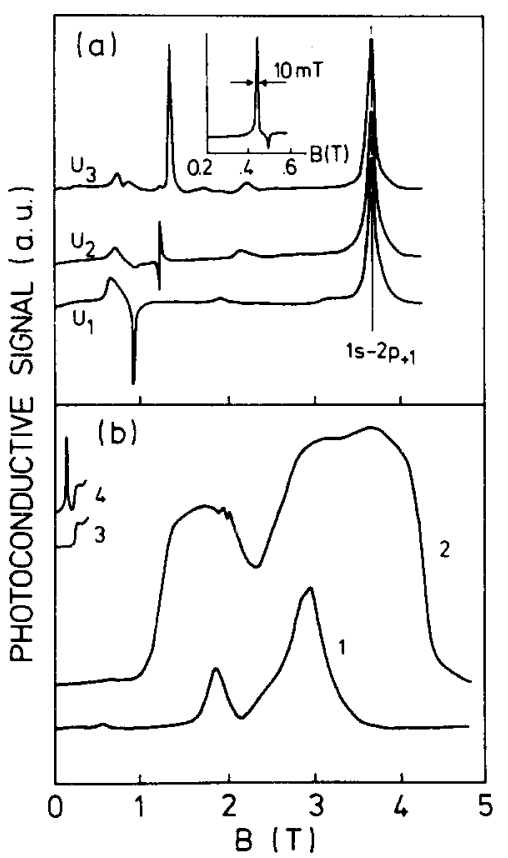

Fig. 3

Magnetophotoconductivity

(a) at $\lambda=118.6 \mu \mathrm{m}$ for various biasing conditions; $U_{1}=4.6 \mathrm{~V}, U_{2}=4.9 \mathrm{~V}, U_{3}=5.0 \mathrm{~V}$, Inset $I=$ $2.207 \mathrm{~mA}$

(b) at $\lambda=337 \mu \mathrm{m}$; constant bias current (1) and (2) $3.096 \mathrm{~mA},(3)$ and (4) $2.590 \mathrm{~mA}$; the intensities of measurements (2) and (4) were about $25 \mathrm{~mW}$. $\mathrm{cm}^{-2}$ and ten times higher than those of recordings (1) and (3).

processes may increase the photoconductive signal over that of the photo-thermal effect both by raising free carrier generation and by reducing the recombination rate. The generation rate is increased by impact ionization of the optically populated final state [5]. As free carriers are captured in high excited states and cascade down to the ground state by emission of phonons, impact ionization of impurities in excited states generally lowers the recombination rate and thus increases the life time in the conduction band [6].

The spectral structures which are not caused by optical transitions between bound donor states and which shift to higher magnetic fields with increasing bias voltage are very similar to those observed in the magnetoresistance of $\mathrm{n}-\mathrm{GaAs}$ for voltages below the one stage impact ionization instability [7]. The abrupt changes in the electrical resistance were attributed to inelastic scattering of hot electrons by excited neutral donors populated by impact excitation. This conclusion was derived from the scaling of free electron temperature with donor excitation energies and the shift of the structures due to cooling of the electron system in a magnetic field. In contrast to the magnetoresistance effects, the present photoconductivity struc- tures arise well above breakdown of the sample but below saturation of the conduction band with electrons. In this regime of the current-voltage characteristic the current flow is filamentary. Outside the filament in the high-ohmic cladding regions the capture rate of electrons exceeds the impact ionization rate and these portions of the sample are locally below breakdown. In particular in the filament borders a high density of excited donors is present [1] which may be populated by carriers drifting out of the filament with average energy $k_{B} T_{e}=E^{*}-E_{1}$ s where $T_{e}$ is the electron temperature and $E^{*}-E_{1 s}$ is the donor excitation energy. Photoionization out of these excited states may cause the observed effects. Increasing the magnetic field cools the electron gas, therefore higher bias voltages are required to obtain the necessary electron temperature. Within this interpretation the spectral structures shown in Fig. 2a and 2b at both sides of the $1 s-2 p_{+1}$ line and the cyclotron resonance, respectively, must be due to excited donor states of long life time $[8,9,10]$.

The observed large signal strengths and the intensity dependence of the photosignal, however, cannot be explained by assuming linear photoconductivity in the filament borders. A current filament is a dissipative structure which is formed by a critical balance of outward and inward directed forces on the carriers. Structure forming forces on the current flow in semiconductor are the internal pressure $n k_{B} T_{e}$ where $n$ is the electron density, lateral diffusion, drift by local electric fields, and the self attraction of a current [2]. By balancing these forces the electron temperature in a filament may be estimated. Within the framework of a recently developed model of filament formation in thin semiconductor films, $T_{\mathrm{e}} \approx 20 \mathrm{~K}$ is obtained for $1 \mathrm{~mA}$ at weak magnetic films [11]. This value is in good agreement to the electron temperature derived from magnetoresistance measurements under similar biasing conditions [7]. Outside the filament $T_{e}$ is practically equal to the lattice temperature. Slight redistribution of the electron population by infrared irradiation disturbs the delicate balance of the filament forming processes and may lead to large changes in the overall current through the sample. Optical generation of a small number of free carriers in the filament borders tend to widen the filament causing an overproportional increase of the current which is recorded as a photoconductive signal.

Finally we note that a current filament generated by impact ionization represents a state far from thermal equilibrium and population inversion in the donor energy levels may occur. This might explain the phase changes observed in the center of the $1 s-2 p_{+1}$ line as shown in Fig. 1. Again not a linear effect but a dynamical phenomenon due to a spatial rearrangement of the whole current filament is expected. Outside the filament free electrons are generated whereas inside the filament the electron density is somewhat reduced due to an enhancement of recombination caused by stimulated emission. This lowers the lateral electron diffusion and the self attraction of the current which is pro- 
portional to the current density in the filament. These effects are counteracting as diffusion tends to widen the filament width whereas pinching shrinks the filament. Thus the outcome depends sensitively on the carrier kinetics and both a dip in the line center as well as an enhancement of the signal are conceivable.

In summary pronounced spectral structures have been observed by far-infrared magneto-photoconductivity in high-purity $n-G a A s$ epitaxial layers in a state far from thermal equilibrium. The physical mechanisms leading to these structures are by far not well understood. All experimental results point to dynamical effects induced by infrared irradiation which infringe the self organization of current filaments.

\section{Acknowledgement}

W.P. thanks the Deutsche Forschungsgemeinschaft for financial support.
[1] A. Brandl, M. Völker, and W. Prettl, Appl. Phys. Lett. 55, 238 (1989).

[2] A. Brandl, W. Kröninger, W. Prettl, and G. Obermair, Phys. Rev. Lett. 64, 212 (1990).

[3] V.G. Golubev, V.I. Ivanov-Omskii, A.V. Osutin, R.P. Seisyan, Al.L. Efros, and T.V. Yazeva, Sov. Phys. Semicond. 22, 896 (1988).

[4] H.P. Wagner and W. Prettl, Sol. State Commun. 66, 367 (1988).

[5] G. Jungwirt, G. Kropf and W. Prettl, Int. J. Infrared and Millimeter Waves, to be published.

[6] G. Jungwirt and W. Prettl, Infrared Physics 32, 191 (1991).

\section{References}

[7] S.N. Holmes, P.D. Wang, D.A. Cowan, C. Trager, and R.A. Stradling, Semicond. Sci. Technol. 5, 150 (1990).

[8] V.N. Zverev and D.V. Skovkun, Sov. Phys. JETP 60, 1003 (1984).

[9] J. Kaminski, J. Spector, W. Prettl, and M. Weispfenning, Appl. Phys. Lett. 52, 233 (1988).

[10] V.N. Gantmakher and V.N. Zverev in Landau Level Spectroscopy, eds. G. Landwehr and E.I. Rashba, Elsevier 1991.

[11] A. Brandl and W. Prettl, Phys. Rev. Lett. 66, 3044 (1991). 